\title{
Five Genes Associated With Survival in Patients With Lower-grade Gliomas Were Identified by Information-theoretical Analysis
}

\author{
KEIKO SATO ${ }^{1}$, KOUJI TAHATA ${ }^{1}$ and KAZUNORI AKIMOTO ${ }^{2}$ \\ ${ }^{1}$ Department of Information Sciences, Faculty of Science and Technology, \\ Tokyo University of Science, Chiba, Japan; \\ ${ }^{2}$ Department of Medicinal and Life Science, Faculty of Pharmaceutical Sciences, \\ Tokyo University of Science, Chiba, Japan
}

\begin{abstract}
Background/Aim: Understanding of the molecular events associated with progression and survival differences in patients with lower-grade gliomas (LGGs) is still unclear. The comparison of findings across studies using different datasets and methods is essential for a new molecular-based classification system. The aim of the study was to identify biomarkers for prognostic classification of patients with LGGs, and furthermore to lay a foundation for future development of targeted therapies for LGGs. Patients and Methods: Using information-theoretic and statistical approaches, we analyzed $m R N A$ expression data for 18,413 genes from LGG samples in order to identify candidate biomarkers for survival. The candidate genes were then evaluated for their potential as prognostic biomarkers using multivariable Cox regression analyses that adjusted for the effects of age and grade. Results: WEE1, EMP3, E2F7, CD58 and NSUN7 genes were identified as candidate biomarkers of LGGs and their high expression was associated with significantly shorter survival. The hazard ratios for mortality were 5.02 (95\% CI=3.40-7.40) for WEE1, 5.45 (95\% CI=3.63-8.18) for EMP3, 4.49 (95\% CI=3.03-6.66) for E2F7, 4.77 (95\% CI=3.22-7.06) for CD58 and 4.38 (95\% $C I=2.97-6.47)$ for NSUN7. In addition, the expression pattern of these genes, associated with shorter survival in LGGs, was also observed in glioblastoma multiforme. Conclusion: Identification of genes associated with poor outcomes will provide insights into
\end{abstract}

This article is freely accessible online.

Correspondence to: Keiko Sato, Department of Information Sciences, Faculty of Science and Technology, Tokyo University of Science, 2641 Yamazaki, Noda, Chiba 278-8510, Japan. Tel.: +81 471241501, e-mail: keiko@is.noda.tus.ac.jp

Key Words: WEE1, EMP3, E2F7, CD58, NSUN7, lower-grade gliomas, information-theoretical approach. novel biological mechanisms that may lead to improvement in progression and survival for patients with LGGs.

Low-grade and intermediate-grade gliomas (WHO grades II and III) are less aggressive tumors than high-grade gliomas (WHO grade IV) called glioblastoma multiforme (GBM). Combined grade II and III gliomas have been commonly referred to as lower-grade gliomas (LGGs) (1-3). LGG patients have a better prognosis and longer survival than GBM patients; however, the outcomes of LGG patients are variable; some progress to GBM within months, while others progress slower or remain stable for years $(1,4,5)$. Over the years, numerous attempts have been made to identify prognostic biomarkers and to improve survival in LGG patients. Notably, isocitrate dehydrogenase (IDH) mutation status has been recognized as a prognostic biomarker in gliomas (6-8) and has been included in the current WHO 2016 classification (9). However, as regards the prognostic significance of IDH mutation in LGGs, inconsistent results have been reported $(4,10-15)$. Understanding of the molecular events associated with the progression and survival differences in these patients is an active area of research. The comparison of findings across studies using different datasets and methods is vital for the development of a clinically applicable molecularbased classification system and new targeted therapies as well. At the same time, determining how to handle a huge amount of molecular and clinical data and then how to extract clinically meaningful targets is a significant challenge, not only for gliomas but also for tumors in general.

Herein, we applied an information-theoretic approach to the comprehensive analysis of gene expression patterns in order to identify biomarkers for prognostic classification of LGG patients, and furthermore to lay a foundation for future development of targeted therapies for LGGs. In a previous paper, we showed that the similar information-theoretic analysis is an effective and powerful tool for identifying prognostic factor for survival in patients with estrogen 
Table I. Clinical characteristics of the sample datasets.

\begin{tabular}{lcc}
\hline Characteristic & LGGs, $n(\mathrm{n}=530)$ & GBM, $\mathrm{n}(\mathrm{n}=166)$ \\
\hline Age $^{1}$, years & $41(14-87)$ & $60(21-89)$ \\
Gender (Female/Male/NA) & $238 / 291 / 1$ & $58 / 107 / 1$ \\
IDH (mutant/wild type/NA) & $127 / 308 / 95$ & $0 / 0 / 166$ \\
Grade (II/III/IV/NA) & $258 / 270 / 0 / 2$ & $0 / 0 / 166 / 0 *$ \\
Clinical outcomes & $0-211$ & $0-88$ \\
Overall survival ${ }^{2}$, (months) & 104 & 130 \\
Short-term survival (death<5 years) & 70 & 2 \\
Long-term survival (survival>5 years or censored $>5$ years) & 354 & 32 \\
Censored $<5$ years & 2 & 2 \\
NA & 2 \\
\hline
\end{tabular}

LGGs, Lower-grade gliomas; GBM, glioblastoma multiforme; IDH, isocitrate dehydrogenase. ${ }^{1}$ Data presented as median (range). ${ }^{2}$ Data presented as range. *The grades for GBM cases were not provided in the original TCGA dataset.

receptor-positive breast cancer (16). In information theory, information is quantified using the concept of uncertainty. According to Shannon, information is related to uncertainty in the view that reduction of uncertainty can be regarded as acquisition of information (17). We focus on how the uncertainty of prognosis for LGG patients can be reduced by the acquisition of information on gene expression levels.

\section{Patients and Methods}

Method overview. To better understand molecular events associated with the progression and survival differences in LGGs, we used the 'Brain Lower Grade Glioma' and 'Glioblastoma Multiforme)' datasets of The Cancer Genome Atlas (TCGA, Provisional). Data for the LGG and GBM samples were downloaded on July 27, 2019 from the cBioPortal for Cancer Genomics (http://www.cbioportal.org/) $(18,19)$. Clinical details for these samples are given in Table I. The LGG dataset, consisting of 530 samples, included mRNA expression data for 18,413 genes. We analyzed the mRNA expression z-scores of the LGG subgroup using information-theoretic and statistical approaches to identify candidate biomarkers for survival of LGGs. In addition, we investigated the relationships between LGGs and GBM based on the expression of each gene that was identified as a candidate biomarker for LGGs.

Information-theoretic analysis. For 174 patient samples in the TCGA LGG dataset -356 samples were excluded (354 were censored within 5 years after diagnosis and 2 had no clinical information)- the following two events were defined: $\left(x_{1}\right)$, short-term survival (death within 5 years after diagnosis), and $\left(x_{2}\right)$, long-term survival (survival $>5$ years after diagnosis). The probabilities of these two events are given by $p\left(x_{1}\right)=104 / 174$ and $p\left(x_{2}\right)=70 / 174$, respectively. The Shannon entropy of the complete event system $X=\left(\begin{array}{cc}x_{1} & x_{2} \\ p\left(x_{1}\right) & p\left(x_{2}\right)\end{array}\right)$ is defined as $\mathrm{S}(X)=-\sum_{i=1}^{2} p\left(x_{i}\right) \log p\left(x_{i}\right)$, which gives a measure of the uncertainty of $X$ and takes from a minimum value of 0 to a maximum value of $\log 2=1$ ( $\log$ denotes the binary logarithm throughout our analysis). We used this measure to quantify the uncertainty of prognosis in LGG patients. Moreover, we defined the following two events for the mRNA expression level of a gene: $\left(y_{1}\right)$, high-expression $(\mathrm{Z}$-score $>0)$, and $\left(y_{2}\right)$, low-expression $(\mathrm{Z}$-score $<0)$. The complete event system $Y$ is denoted by the probabilities $p\left(y_{1}\right)$ and $p\left(y_{2}\right)$ of these two events, such that $p\left(y_{1}\right)+p\left(y_{2}\right)=1$, which are calculated using the $\mathrm{z}$-scores for each gene across the 174 samples (“data_RNA_Seq_v2_mRNA_median_Zscores.txt” file in the TCGA LGG dataset). The dataset included mRNA expression data for 18,413 genes. For each gene, the complete event system $Y$ can be determined. The compound event system $X \times Y$ for $X$ and $Y$ is denoted by the joint probability $p\left(x_{i}, y_{j}\right)$ for the event $x_{i}(i=1,2)$ and event $y_{j}(j=1,2)$, which satisfy $\Sigma_{j} p\left(x_{i}, y_{j}\right)=p\left(x_{i}\right)$ and $\Sigma_{i} p\left(x_{i}, y_{j}\right)=p\left(y_{j}\right)$. Using these event systems, the mutual information is defined as as $\Sigma_{i, j} p\left(x_{i}, y_{j}\right) \log \frac{p\left(x_{i}, y_{j}\right)}{p\left(x_{i}\right) p\left(y_{j}\right)}$, which is non-negative. The mutual information measures the reduction in uncertainty of $X$ due to the knowledge of $Y$. Larger mutual information implies less uncertainty in $X$. For each gene, the mutual information was calculated. The genes were then ranked based on the mutual information so that the gene with the largest mutual information was number 1 . This analysis allowed us to quantify how the uncertainty of the prognosis is reduced through the acquisition of information on gene expression levels, as well as to identify candidate prognostic biomarkers for LGGs.

Statistical analysis. In addition to the information-theoretic analysis, for the 174 LGG samples, we used a chi-square test of independence to assess the association between the expression level (high or low) of each gene for the 18,413 expressed genes and the overall survival (short or long). Thus, we identified candidate genes from both information-theoretic and statistical measures. These candidate genes were then evaluated for their potential as prognostic biomarkers in LGGs. For the 528 LGG samples, including 354 samples censored within 5 years after diagnosis, the Kaplan-Meier method was used to calculate survival rates, and log-rank testing was applied to compare survival differences between expression status. Univariable and multivariable Cox proportional hazards models were used to estimate hazard ratios and $95 \%$ confidence intervals for mortality. Univariable analysis was performed for each of the candidate genes and other prognostic variables, including age, gender, IDH1 mutation status and grade, and those variables that were significant $(p<0.05)$ were included in multivariable analysis. 
Table II. Top 10 genes having potential relevance to the progression and survival differences in lower-grade gliomas.

\begin{tabular}{|c|c|c|c|c|c|}
\hline \multicolumn{3}{|c|}{ Information-theoretic analysis* } & \multicolumn{3}{|c|}{ Chi-square analysis } \\
\hline Gene & Mutual information & $\begin{array}{c}\text { Ranking } \\
\text { (chi-square analysis) }\end{array}$ & Gene & $\begin{array}{l}\text { Chi-square } \\
\text { value }\end{array}$ & $\begin{array}{c}\text { Ranking } \\
\text { (information-theoretic analysis) }\end{array}$ \\
\hline WEE1 & 0.2578 & 1 & WEE1 & 57.00 & 1 \\
\hline EMP3 & 0.2190 & 4 & E2F7 & 44.32 & 3 \\
\hline E2F7 & 0.2070 & 2 & CD58 & 41.54 & 4 \\
\hline CD58 & 0.2005 & 3 & EMP3 & 41.37 & 2 \\
\hline NSUN7 & 0.1933 & 5 & NSUN7 & 39.15 & 5 \\
\hline DMRTA2 & 0.1912 & 31 & $\mathrm{EVC} 2$ & 38.89 & 8 \\
\hline LNC00669 & 0.1886 & 11 & ALG6 & 38.22 & 19 \\
\hline $\mathrm{EVC} 2$ & 0.1878 & 6 & TNFAP6 & 37.88 & 9 \\
\hline TNFAP6 & 0.1871 & 8 & PTGFRN & 37.61 & 17 \\
\hline PDPN & 0.1765 & 21 & TMEM220 & 37.45 & 16 \\
\hline
\end{tabular}

*The Shannon entropy, $\mathrm{S}(X)=0.9722$.

Furthermore, we investigated the relationships between LGG and GBM samples based on the expression level of each gene that was identified as a candidate biomarker for LGGs, using data (data_RNA_Seq_v2_expression_median.txt) from the two TCGA datasets (530 LGG samples and 166 GBM samples). All statistical analyses were performed using JMP 14 (SAS Institute Inc. Cary, $\mathrm{NC}$, USA), except for the chi-square test for independence which was calculated directly from the number of occurrences of each compound event in the information-theoretic analysis.

\section{Results}

The 18,413 genes were sorted in descending order based on their mutual information, and similarly, they were sorted according to their chi-square values. The results for the top 10 genes from each analysis are shown in Table II. Of the top 10 genes selected according to the information-theoretic analysis, 7 were also ranked in the top 10 by the chi-square analysis. The top 5 genes were common in both analyses, although the order of ranking differed. Therefore, these five genes (WEE1, EMP3, E2F7, CD58 and NSUN7) were identified as candidate biomarkers for survival of LGGs. We observed that among the 83, 49, 67, 59 and 54 LGG samples with high expression of WEE1, EMP3, E2F7, CD58 and NSUN7 respectively, 74 cases $(89.16 \%)$ for WEE1, 48 cases $(97.96 \%)$ for EMP3, 61 cases $(91.04 \%)$ for E2F7, 55 cases $(93.22 \%)$ for CD58 and 51 cases $(94.44 \%)$ for NSUN7 died within 5 years.

In the survival curves shown in Figure 1, the 528 LGG samples, including 354 samples censored within 5 years after diagnosis, were divided into the two groups based on the expression level $(\mathrm{Z}$-score $>0$ or $\mathrm{Z}$-score $<0)$ of each gene. High expression levels of WEE1, EMP3, E2F7, CD58 and NUSN7 were associated with shorter survival in LGGs. For WEE1, the 5-year survival rate was $21.8 \%$ in high expression (+) versus $79.1 \%$ in low expression (-); for
Table III. Multivariable analysis of prognostic factors associated with survival differences in lower-grade gliomas (LGGs).

\begin{tabular}{|c|c|c|}
\hline \multirow[t]{2}{*}{ Variable } & \multicolumn{2}{|c|}{ LGGs $\left(\mathrm{n}=527^{*}\right)$} \\
\hline & $\mathrm{HR}(95 \% \mathrm{CI})$ & $p$-Value \\
\hline \multicolumn{3}{|l|}{ Age at diagnosis } \\
\hline$>40 / \leq 40$ years & $2.97(2.01-4.45)$ & $<0.0001$ \\
\hline \multicolumn{3}{|l|}{ Grade } \\
\hline III/II & $1.73(1.15-2.65)$ & 0.0078 \\
\hline \multicolumn{3}{|c|}{ WEE1 expression level } \\
\hline High $(+) /$ low $(-)$ & $5.02(3.40-7.40)$ & $<0.0001$ \\
\hline \multicolumn{3}{|l|}{ Age at diagnosis } \\
\hline$>40 / \leq 40$ years & $2.09(1.40-3.15)$ & 0.0003 \\
\hline \multicolumn{3}{|l|}{ Grade } \\
\hline $\mathrm{III} / \mathrm{II}$ & $2.29(1.54-3.45)$ & $<0.0001$ \\
\hline \multicolumn{3}{|c|}{ EMP3 expression level } \\
\hline High $(+) /$ low $(-)$ & $5.45(3.63-8.18)$ & $<0.0001$ \\
\hline \multicolumn{3}{|l|}{ Age at diagnosis } \\
\hline$>40 / \leq 40$ years & $2.51(1.71-3.74)$ & $<0.0001$ \\
\hline \multicolumn{3}{|l|}{ Grade } \\
\hline $\mathrm{III} / \mathrm{II}$ & $1.89(1.24-2.92)$ & 0.0030 \\
\hline \multicolumn{3}{|c|}{ E2F7 expression level } \\
\hline High (+)/low (-) & $4.49(3.03-6.66)$ & $<0.0001$ \\
\hline \multicolumn{3}{|l|}{ Age at diagnosis } \\
\hline$>40 / \leq 40$ years & $2.51(1.71-3.72)$ & 0.0003 \\
\hline \multicolumn{3}{|l|}{ Grade } \\
\hline $\mathrm{III} / \mathrm{II}$ & $2.32(1.56-3.51)$ & $<0.0001$ \\
\hline \multicolumn{3}{|c|}{ CD58 expression level } \\
\hline High $(+) /$ low $(-)$ & $4.77(3.22-7.06)$ & $<0.0001$ \\
\hline \multicolumn{3}{|l|}{ Age at diagnosis } \\
\hline$>40 / \leq 40$ years & $2.68(1.82-4.01)$ & $<0.0001$ \\
\hline \multicolumn{3}{|l|}{ Grade } \\
\hline $\mathrm{III} / \mathrm{II}$ & $2.08(1.40-3.14)$ & 0.0002 \\
\hline \multicolumn{3}{|c|}{ NSUN7 expression level } \\
\hline High $(+) /$ low $(-)$ & $4.38(2.97-6.47)$ & $<0.0001$ \\
\hline
\end{tabular}

HR, Hazard ratio; CI, confidence interval. *Of the 530 LGG samples, 3 cases did not have available overall survival and grade information. 
A

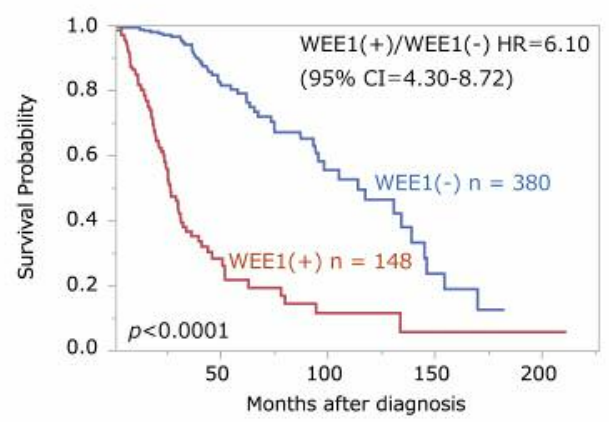

C

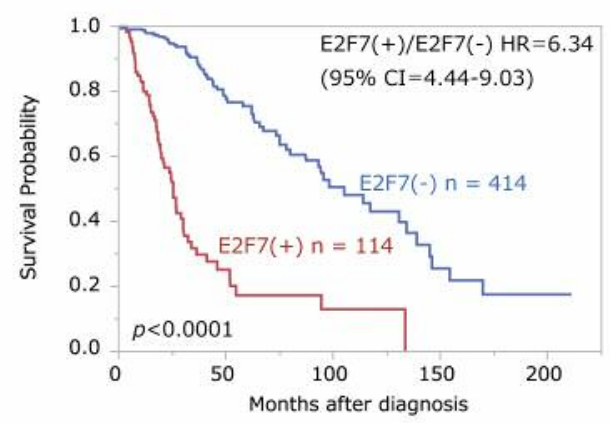

E

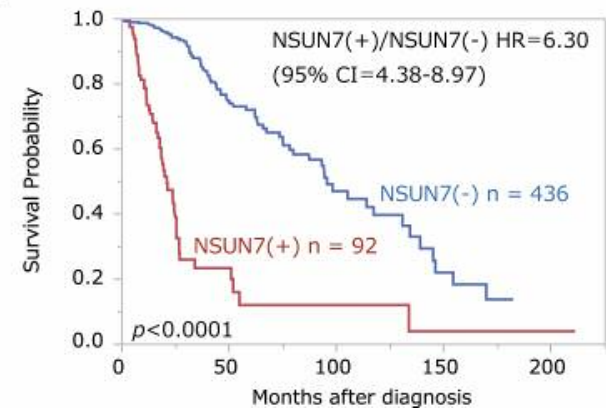

EMP3, it was $5.1 \%(+)$ versus $71.8 \%(-)$; for E2F7, it was $17.2 \%(+)$ versus $75.3 \%(-)$; for CD58, it was $17.5 \%(+)$ versus $73.0 \%(-)$; for NUSN7, it was $12.0 \%(+)$ versus $72.0 \%(-)$. In each case, the differences were significant $(p<0.0001)$. The univariable hazard ratio (HR) was 6.10 [95\% confidence interval $(\mathrm{CI})=4.30-8.72]$ for WEE1, 8.70 (95\% CI=5.93-12.65) for EMP3, 6.34 (95\% CI=4.44-9.03) for E2F7, 6.62 (95\% CI=4.56-9.60) for CD58 and $6.30(95 \%$ $\mathrm{CI}=4.38-8.97)$ for NUSN7.

In addition, there were significant differences in survival based on age or grade. The 5-year survival rate was $46.8 \%$ in LGG subgroup $>40$ years and $75.3 \%$ in those $\leq 40$ years $(p<0.0001)$. The 5 -year survival rate was $42.8 \%$ in LGG subgroup with grade III and $81.0 \%$ in those with grade II $(p<0.0001)$. The HR was 2.90 for age $(>40 / \leq 40)(95 \%$
B

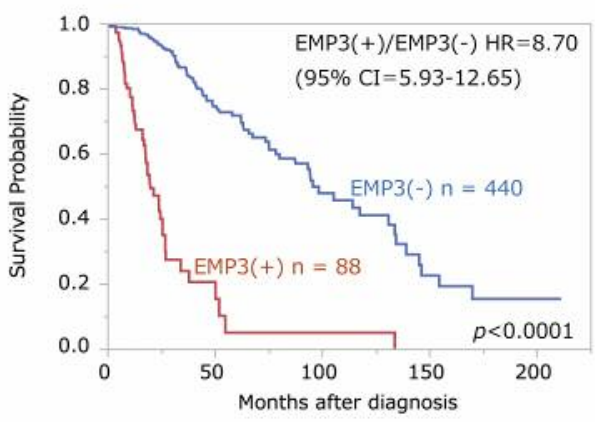

D

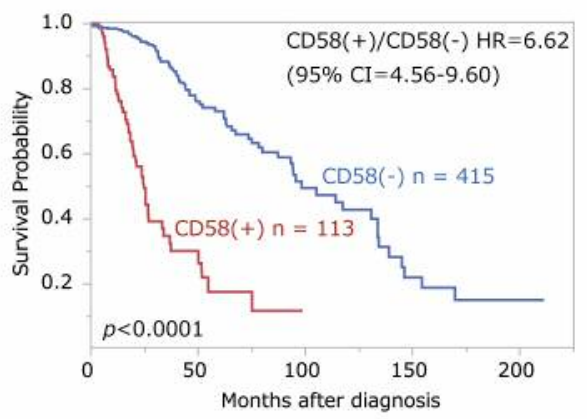

Figure 1. Survival curves for the two lower-grade glioma ( $L G G)$ groups based on the expression level [Z-score $>0(+)$ or Z-score $<0(-)]$ of each gene, (A) WEE1, (B) EMP3, (C) E2F7, (D) CD58 and (E) NSUN7. pValues were calculated using the log-rank test. Hazard ratio $(H R)$ and 95\% confidence interval (CI) were calculated from Cox proportional hazards models.

$\mathrm{CI}=2.01-4.23)$ and 3.35 for grade (III/II) $(95 \% \mathrm{CI}=2.31-4.94)$ in univariable analysis. However, significant differences in survival were not observed based on gender or IDH1 mutation status. The 5-year survival rate was $65.0 \%$ in LGG subgroup of women and $59.0 \%$ in those of men $(p=0.4482)$. The 5-year survival rate was $59.4 \%$ in LGG subgroup with IDH1 mutations and $62.1 \%$ in those without IDH1 mutations $(p=0.6818)$. The HR was 0.87 for gender (female/male) (95\% CI=0.61-1.23) and 0.90 for IDH1 (mutant/wild type) (95\% $\mathrm{CI}=0.53-1.45)$ in univariable analysis.

Multivariable analysis also indicated that age, grade, and each of the 5 candidate genes (WEE1, EMP3, E2F7, CD58 and NSUN7) were significantly associated with differences in survival for LGGs (Table III). Above all, high expression of these genes in LGGs was the main risk factor for 
A

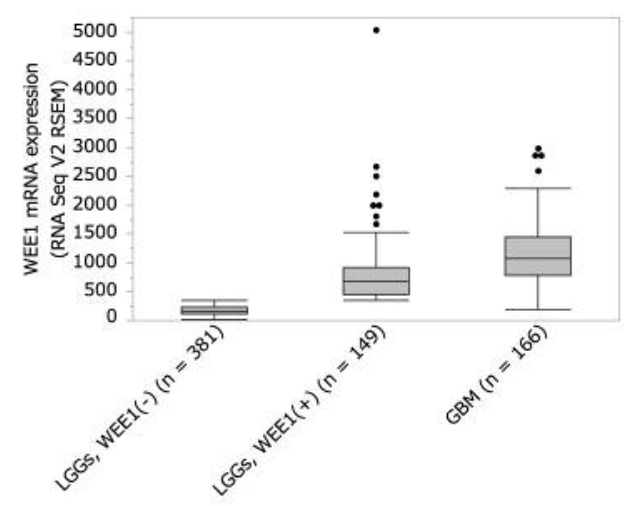

C

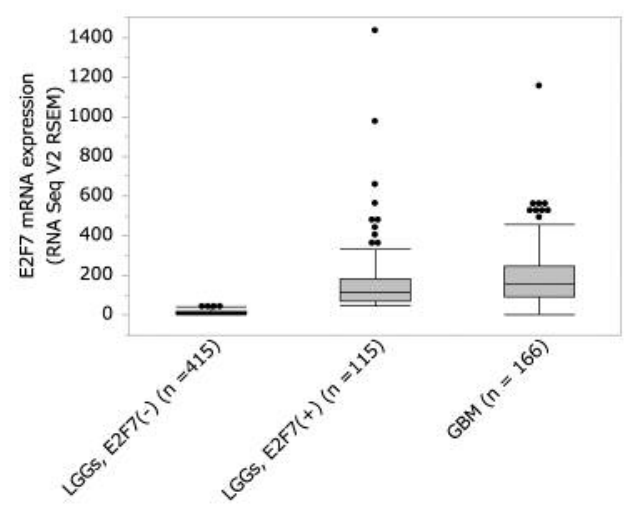

E

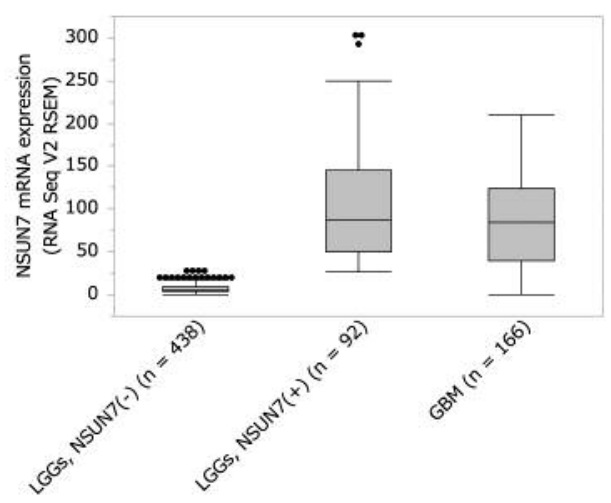

increased mortality. The HR adjusted for age and grade was $5.02(95 \% \mathrm{CI}=3.40-7.40 ; p<0.0001)$ for WEE1, $5.45(95 \%$ $\mathrm{CI}=3.63-8.18 ; p<0.0001)$ for $\mathrm{EMP} 3,4.49(95 \% \mathrm{CI}=3.03-$ $6.66 ; p<0.0001)$ for $\mathrm{E} 2 \mathrm{~F} 7,4.77(95 \% \mathrm{CI}=3.22-7.06$; $p<0.0001)$ for $\mathrm{CD} 58$ and $4.38 \quad(95 \% \quad \mathrm{CI}=2.97-6.47$; $p<0.0001)$ for NSUN7.

The pattern of high mRNA expression of these genes associated with shorter term survival in LGGs was also
B

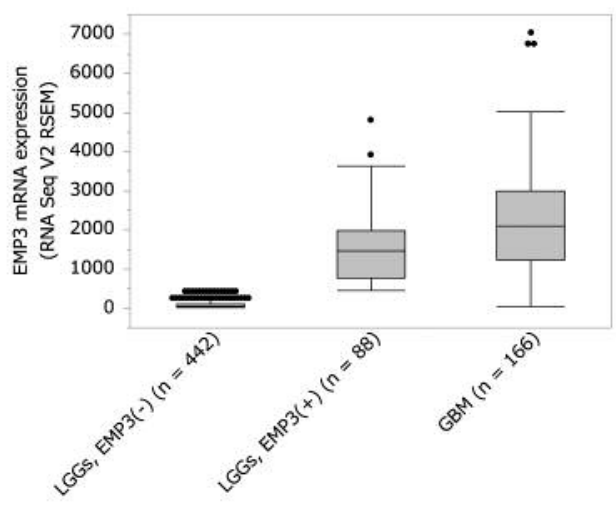

D

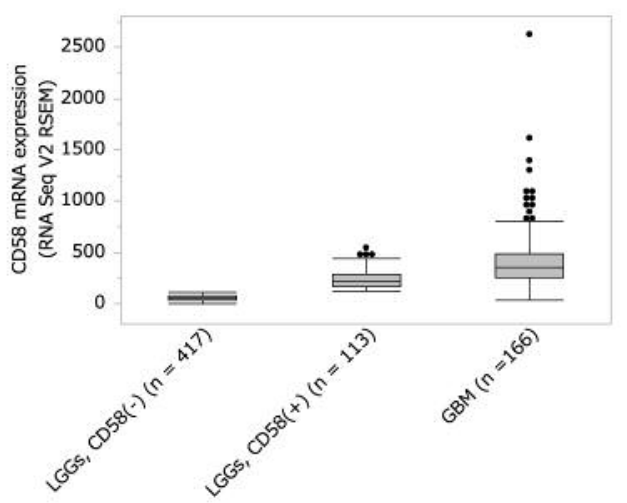

Figure 2. Comparison of mRNA levels of WEE1 (A), EMP3 (B), E2F7(C), CD58 (D) and NSUN7 (E) in the two lower-grade glioma $(L G G)$ groups based on the expression level [Z-score $>0(+)$ or $Z$ score $<0(-)]$ of each gene and the glioblastoma multiforme (GBM) group. Data are shown as box plots. Each box represents the 25th to 75 th percentiles. The horizontal line in each box represents the median mRNA level in each group.

observed in 166 GBM samples (Figure 2). The respective median values in the GBM group were nearly equal to or higher than those in the LGG subgroup with high expression. In any case, the respective median values in LGG subgroup with low expression were quite low compared to those in the GBM group. These results indicate that high mRNA levels of WEE1, EMP3, E2F7, CD58 and NSUN7 are linked to poor survival in gliomas. 
A

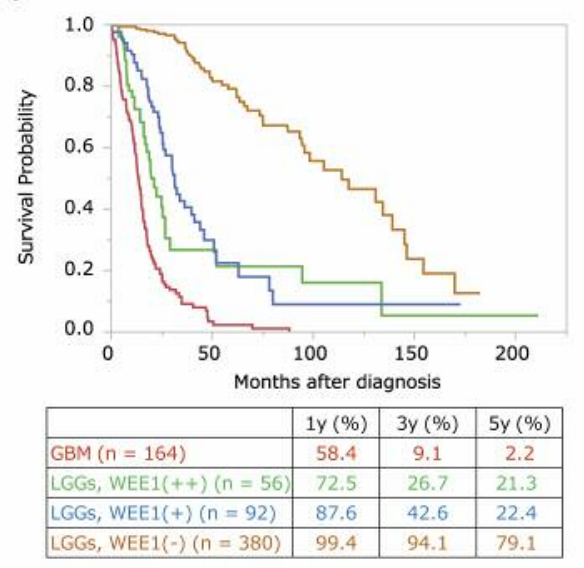

C

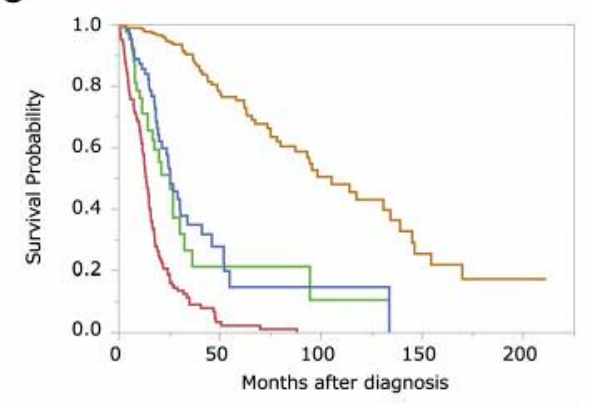

\begin{tabular}{|l|c|c|c|}
\hline & $1 y(\%)$ & $3 y(\%)$ & $5 y(\%)$ \\
\hline GBM $(n=164)$ & 58.4 & 9.1 & 2.2 \\
\hline LGGs, E2F7 $(++)(n=44)$ & 71.0 & 26.5 & 21.2 \\
\hline LGGs, E2F7 $(+)(n=70)$ & 85.6 & 34.9 & 14.8 \\
\hline LGG5, E2F7 $(-)(n=414)$ & 98.6 & 90.3 & 75.3 \\
\hline
\end{tabular}

E

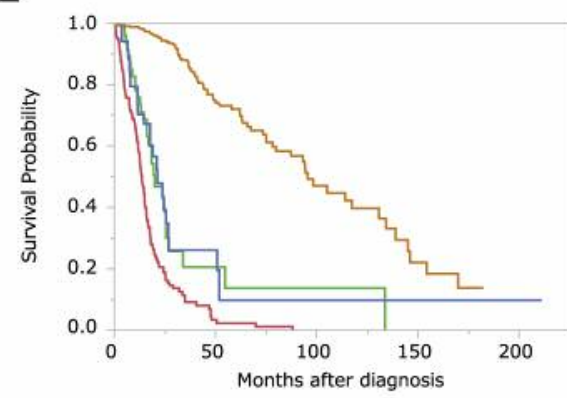

\begin{tabular}{|l|c|c|c|}
\hline & $1 y(\%)$ & $3 y(\%)$ & $5 y(\%)$ \\
\hline GBM $(n=164)$ & 58.4 & 9.1 & 2.2 \\
\hline LGGs, NSUN7 $(++)(n=51)$ & 75.8 & 20.5 & 13.7 \\
\hline LGGs, NSUN7 $(+)(n=41)$ & 70.2 & 26.1 & 9.7 \\
\hline LGGs, NSUN7 $(-)(n=436)$ & 98.7 & 88.0 & 72.0 \\
\hline
\end{tabular}

For each gene, the LGG samples with high expression were further divided into two subgroups according to the expression level: Z-score $\geq 1 \quad(++)$ and $0<Z$-score $<1 \quad(+)$ (Figure 3). Although there was a significant difference in survival between the CD58(++) and CD58(+) subgroups ( $p=0.0001$ by log-rank test), no significant differences were
B

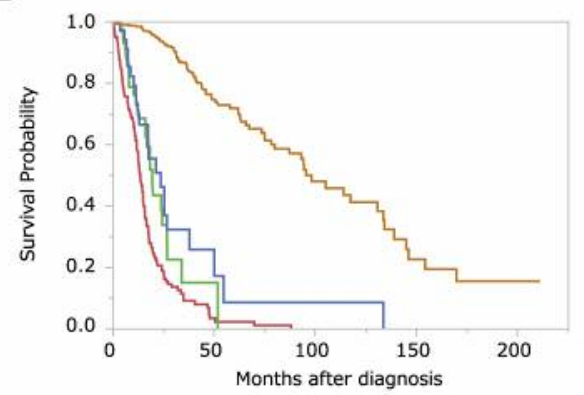

\begin{tabular}{|l|c|c|c|}
\hline & $1 y(\%)$ & $3 y(\%)$ & $5 y(\%)$ \\
\hline GBM $(n=164)$ & 58.4 & 9.1 & 2.2 \\
\hline LGGs, EMP3 $(++)(n=50)$ & 73.7 & 15.0 & 0 \\
\hline LGGs, EMP3 $(+)(n=38)$ & 72.8 & 32.3 & 8.6 \\
\hline LGGs, EMP3 $(-)(n=440)$ & 98.5 & 86.7 & 71.8 \\
\hline
\end{tabular}

D

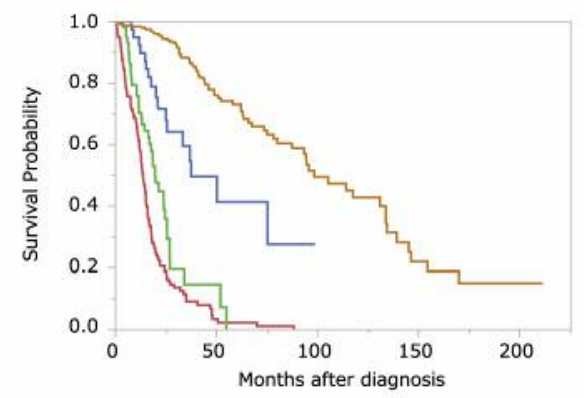

\begin{tabular}{|l|c|c|c|}
\hline & $1 y(\%)$ & $3 y(\%)$ & $5 y(\%)$ \\
\hline GBM $(n=164)$ & 58.4 & 9.1 & 2.2 \\
\hline LGGs, CD58(++) (n=67) & 70.3 & 14.6 & 0 \\
\hline LGGs, CD58(+) (n=46) & 92.3 & 59.5 & 41.3 \\
\hline LGGs, CD58(-) (n=415) & 98.4 & 88.3 & 73.0 \\
\hline
\end{tabular}

Figure 3. Survival curves for the three lower-grade glioma (LGG) groups based on the expression level [Z-score $\geq 1(++), 0<Z$-score $<1$ (+) or Z-score $<0$ (-)] of each gene, (A) WEE1, (B) EMP3, (C) E2F7, (D) CD58 and $(E)$ NSUN7. As a reference, the corresponding curve for the GBM group is also depicted. Of the 166 glioblastoma multiforme (GBM) samples, 2 cases did not have available clinical information. The 1-, 3-, and 5-year survival rates were calculated using the KaplanMeier method.

observed between the subgroups with different expression levels of WEE1, EMP3, E2F7 and NSUN7 $(p=0.0794$, $p=0.4210, p=0.8687$ and $p=0.8474$, respectively).

Interestingly, for the LGG(+) group consisting of $54 \mathrm{LGG}$ samples (grade II, $\mathrm{n}=3$; and grade III, $\mathrm{n}=51$ ) with high expression (Z-score $>0)$ of all five genes, the 1-, 3- and 5- 
year survival rates were $64.2 \%, 5.6 \%$ and $0 \%$, respectively, while for the LGG(-) group consisting 330 LGG samples (grade II, $\mathrm{n}=211$; grade III, $\mathrm{n}=118$; and NA, $\mathrm{n}=1$ ) with low expression $(\mathrm{Z}$-score $<0)$ of all five genes, the 1-, 3- and 5year rates were $99.3 \%, 95.5 \%$ and $83.2 \%$, respectively. There was a significant difference in survival between the two groups $(p<0.0001$ by log-rank test). The corresponding survival rates for the GBM group were $58.4 \%, 9.1 \%$ and $2.2 \%$, respectively.

\section{Discussion}

Information-theoretic and statistical approaches contributed to the identification of WEE1, EMP3, E2F7, CD58 and NSUN7 genes as potential prognostic biomarkers and high expression of these genes was associated with significantly shorter survival in LGGs.

WEE1 is a tyrosine kinase, which has been shown to act as a regulator of the G2 checkpoint in GBM cells $(20,21)$. Inhibition of this kinase has been indicated to enhance sensitivity to irradiation and temozolomide (20-22). Previous studies have revealed that WEE1 is expressed in all glioma types and grades; while, it is overexpressed in GBM (20-22). Shu et al. demonstrated that WEE1 was an independent prognostic biomarker for high-grade gliomas (23).

EMP3 is a transmembrane protein that is involved in cell proliferation and cell-cell interactions, and functions as a tumor suppressor for gliomas $(24,25)$. Ernst et al. have shown that high EMP3 expression was significantly associated with shorter overall survival in astrocytic glioma patients in univariable analysis (26).

E2F7, a member of E2F transcriptional factor, plays an essential role in the regulation of cell cycle progression (27). E2F7 has been reported to be highly expressed in high-grade gliomas compared to low-grade gliomas (28). However, the association between E2F7 expression and overall survival in gliomas has been little studied.

CD58 is a member of the immunoglobulin superfamily, which is a ligand of the $\mathrm{T}$ lymphocyte $\mathrm{CD} 2$ protein, and functions in adhesion and activation of T lymphocytes (29). CD58 protein is a cell-surface marker as cancer stem cell of colorectal cancer (30). Although there is a study showing differences in CD58 expression level between gliomas and normal brain using small number of samples (31), few studies have reported the involvement of CD58 in the progression of gliomas.

NSUN7 belongs to the methyltransferase superfamily and is essential for sperm motility $(32,33)$. To our knowledge, no studies have been conducted on the impact of NUSN7 status on the progression in gliomas.

Within our study, there was no significant difference in survival based on IDH1 mutation status, although IDH mutation status has been recognized as a prognostic biomarker for overall survival in gliomas (6-8). More detailed studies are required to unequivocally determine whether IDH1 mutation status is a prognostic biomarker for LGGs. Nevertheless, our results suggested that the expression levels of WEE1, EMP3, E2F7, CD58 and NSUN7 genes may serve as independent prognostic factors for overall survival in patients with LGGs. Identification of genes associated with poor outcomes will provide insights into novel biological mechanisms that lead to improvement in progression and survival for LGG patients, and lay the foundation for development of novel therapeutic approaches for LGGs. The information-theoretic approach presented here may be an effective and powerful tool for highlighting clinically important genes and can be applied to other tumors.

\section{Conflicts of Interest}

The Authors declare no conflicts of interest.

\section{Authors' Contributions}

K.S. contributed to the conception and design of the study, acquisition of data, analysis and interpretation of data, and drafting and critical revision of the manuscript. K.T. and K.A. contributed to analysis and interpretation of data, and critical revision of the manuscript.

\section{Acknowledgements}

The Authors wish to thank Ms. Mika Kikuno, a former member of the Sato laboratory, for her contribution in the early stages of this work.

\section{References}

1 Zhou H, Vallières M, Bai HX, Su C, Tang H, Oldridge D, Zhang Z, Xiao B, Liao W, Tao Y, Zhou J, Zhang P and Yang L: MRI features predict survival and molecular markers in diffuse lowergrade gliomas. Neuro Oncol 19(6): 862-870, 2017. PMID: 28339588. DOI: $10.1093 /$ neuonc/now256

2 Kiran M, Chatrath A, Tang X, Keenan DM and Dutta A: A prognostic signature for lower grade gliomas based on expression of long non-coding RNAs. Mol Neurobiol 56(7): 4786-4798, 2019. PMID: 30392137. DOI: 10.1007/s12035-018-1416-y

3 Delfanti RL, Piccioni DE, Handwerker J, Bahrami N, Krishnan A, Karunamuni R, Hattangadi-Gluth JA, Seibert TM, Srikant A, Jones KA, Snyder VS, Dale AM, White NS, McDonald CR and Farid N: Imaging correlates for the 2016 update on WHO classification of grade II/III gliomas: implications for IDH, 1p/19q and ATRX status. J Neurooncol 135(3): 601-609, 2017. PMID: 28871469. DOI: 10.1007/s11060-017-2613-7

4 Brat DJ, Verhaak RG, Aldape KD, Yung WK, Salama SR, Cooper LA, Rheinbay E, Miller CR, Vitucci M, Morozova O, Robertson AG, Noushmehr H, Laird PW, Cherniack AD, Akbani R, Huse JT, Ciriello G, Poisson LM, Barnholtz-Sloan JS, Berger MS, Brennan C, Colen RR, Colman H, Flanders AE, Giannini C, Grifford M, Iavarone A, Jain R, Joseph I, Kim J, Kasaian K, Mikkelsen T, Murray BA, O'Neill BP and Pachter L et al: 
Comprehensive, integrative genomic analysis of diffuse lowergrade gliomas. N Engl J Med 372(26): 2481-2498, 2015. PMID: 26061751. DOI: 10.1056/NEJMoa1402121

5 Akkus Z, Ali I, Sedlář J, Agrawal JP, Parney IF, Giannini C and Erickson BJ: Predicting deletion of chromosomal arms 1p/19q in low-grade gliomas from MR images using machine intelligence. J Digit Imaging 30(4): 469-476, 2017. PMID: 28600641. DOI: 10.1007/s10278-017-9984-3

6 Guo C, Pirozzi CJ, Lopez GY and Yan H: Isocitrate dehydrogenase mutations in gliomas: mechanisms, biomarkers and therapeutic target. Curr Opin Neurol 24(6): 648-52, 2011. PMID: 22002076. DOI: 10.1097/WCO.0b013e32834cd415

7 Picca A, Berzero G, Di Stefano AL and Sanson M: The clinical use of IDH1 and IDH2 mutations in gliomas. Expert Rev Mol Diagn 18(12): 1041-1051, 2018. PMID: 30427756. DOI: 10.1080/14737159.2018.1548935.

8 Fukunaga T, Fujita Y, Kishima H and Yamashita T: Methylation dependent down-regulation of G0S2 leads to suppression of invasion and improved prognosis of IDH1-mutant glioma. PLoS One 13(11): e0206552, 2018. PMID: 30388142. DOI: 10.1371/journal.pone.0206552

9 Louis DN, Perry A, Reifenberger G, von Deimling A, FigarellaBranger D, Cavenee WK, Ohgaki H, Wiestler OD, Kleihues P and Ellison DW: The 2016 World Health Organization classification of tumors of the central nervous system: a summary. Acta Neuropathol 131(6): 803-820, 2016. PMID: 27157931. DOI: 10.1007/s00401-016-1545-1

10 Ahmadi R, Stockhammer F, Becker N, Hohlen K, Misch M, Christians A, Dictus C, Herold-Mende C, Capper D, Unterberg A, von Deimling A, Wick W and Hartmann C: No prognostic value of IDH1 mutations in a series of 100 WHO grade II astrocytomas. J Neurooncol 109(1): 15-22, 2012. PMID: 22528790. DOI: 10.1007/s11060-012-0863-y

11 Iwadate Y, Matsutani T, Hirono S, Ikegami S, Shinozaki N and Saeki N: IDH1 mutation is prognostic for diffuse astrocytoma but not low-grade oligodendrogliomas in patients not treated with early radiotherapy. J Neurooncol 124(3): 493-500, 2015. PMID: 26243269. DOI: 10.1007/s11060-015-1863-5

12 Houillier C, Wang X, Kaloshi G, Mokhtari K, Guillevin R, Laffaire J, Paris S, Boisselier B, Idbaih A, Laigle-Donadey F, Hoang-Xuan K, Sanson M and Delattre JY: IDH1 or IDH2 mutations predict longer survival and response to temozolomide in low-grade gliomas. Neurology 75(17): 1560-1566, 2010. PMID: 20975057. DOI: 10.1212/WNL.0b013e3181f96282

13 Poulen G, Gozé C, Rigau V and Duffau H: Huge heterogeneity in survival in a subset of adult patients with resected, wild-type isocitrate dehydrogenase status, WHO grade II astrocytomas. J Neurosurg 130(4): 1289-1298, 2018. PMID: 29676695. DOI: 10.3171/2017.10.JNS171825

14 Kaminska B, Czapski B, Guzik R, Król SK and Gielniewski B: Consequences of IDH1/2 mutations in gliomas and an assessment of inhibitors targeting mutated IDH proteins. Molecules 24(5): E968, 2019. PMID 30857299. DOI: 10.3390/ molecules 24050968

15 Liu B, Liu J, Liu K, Huang H, Li Y, Hu X, Wang K, Cao H and Cheng Q: A prognostic signature of five pseudogenes for predicting lower-grade gliomas. Biomed Pharmacother 117: 109116, 2019. PMID: 31247469. DOI: 10.1016/j.biopha.2019.109116

16 Sato K and Akimoto K: Expression levels of KMT2C and SLC20A1 identified by information-theoretical analysis are powerful prognostic biomarkers in estrogen receptor-positive breast cancer. Clin Breast Cancer 17(3): e135-e142, 2017. PMID: 27986439. DOI: 10.1016/j.clbc.2016.11.005

17 Ohya $\mathrm{M}$ and Volovich I: Information dynamics and adaptive dynamics. In: Mathematical foundations of quantum information and computation and its applications to nano- and bio-systems. Theoretical and Mathematical Physics. Dordrecht, Springer Netherlands, pp. 251-312, 2011. DOI: 10.1007/978-94-0070171-7_10

18 Cerami E, Gao J, Dogrusoz U, Gross BE, Sumer SO, Aksoy BA, Jacobsen A, Byrne CJ, Heuer ML, Larsson E, Antipin Y, Reva B, Goldberg AP, Sander C and Schultz N: The cBio cancer genomics portal: an open platform for exploring multidimensional cancer genomics data. Cancer Discov 2(5): 401-404, 2012. PMID: 2258887. DOI: 10.1158/2159-8290.CD-12-0095

19 Gao J, Aksoy BA, Dogrusoz U, Dresdner G, Gross B, Sumer SO, Sun Y, Jacobsen A, Sinha R, Larsson E, Cerami E, Sander C and Schultz N: Integrative analysis of complex cancer genomics and clinical profiles using the cBioPortal. Sci Signal 6(269): pl1, 2013. PMID: 23550210. DOI: 10.1126/scisignal.2004088

20 Music D, Dahlrot RH, Hermansen SK, Hjelmborg J, de Stricker $\mathrm{K}$, Hansen S and Kristensen BW: Expression and prognostic value of the WEE1 kinase in gliomas. J Neurooncol 127(2): 381389, 2016. PMID: 26738845. DOI: 10.1007/s11060-015-2050-4

21 Mir SE, De Witt Hamer PC, Krawczyk PM, Balaj L, Claes A, Niers JM, Van Tilborg AA, Zwinderman AH, Geerts D, Kaspers GJ, Peter Vandertop W, Cloos J, Tannous BA, Wesseling P, Aten JA, Noske DP, Van Noorden CJ and Würdinger T: In silico analysis of kinase expression identifies WEE1 as a gatekeeper against mitotic catastrophe glioblastoma. Cancer Cell 18(3): 244-257, 2010. PMID: 20832752. DOI: 10.1016/j.ccr.2010.08.011

22 De Witt Hamer PC, Mir SE, Noske D, Van Noorden CJ and Würdinger T: WEE1 kinase targeting combined with DNAdamaging cancer therapy catalyzes mitotic catastrophe. Clin Cancer Res 17(13): 4200-4207, 2011. PMID: 21562035. DOI: 10.1158/1078-0432.CCR-10-2537

23 Shu C, Wang Q, Yan X and Wang J: Whole-genome expression microarray combined with machine learning to identify prognostic biomarkers for high-grade glioma. J Mol Neurosci 64(4): 491-500, 2018. PMID: 29502292. DOI: 10.1007/s12031018-1049-7

24 Alaminos M, Dávalos V, Ropero S, Setién F, Paz MF, Herranz M, Fraga MF, Mora J, Cheung NK, Gerald WL and Esteller M: EMP3, a myelin-related gene located in the critical 19q13.3 region, is epigenetically silenced and exhibits features of a candidate tumor suppressor in glioma and neuroblastoma. Cancer Res 65(7): 2565-2571, 2005. PMID: 15805250. DOI: 10.1158/0008-5472.CAN-04-4283

25 Fumoto S, Tanimoto K, Hiyama E, Noguchi T, Nishiyama M and Hiyama K: EMP3 as a candidate tumor suppressor gene for solid tumors. Expert Opin Ther Targets 13(7): 811-822, 2009. PMID: 19466912. DOI: 10.1517/14728220902988549

26 Ernst A, Hofmann S, Ahmadi R, Becker N, Korshunov A, Engel F, Hartmann C, Felsberg J, Sabel M, Peterziel H, Durchdewald M, Hess J, Barbus S, Campos B, Starzinski-Powitz A, Unterberg A, Reifenberger G, Lichter P, Herold-Mende C and Radlwimmer B: Genomic and expression profiling of glioblastoma stem celllike spheroid cultures identifies novel tumor-relevant genes associated with survival. Clin Cancer Res 15(21): 6541-6550, 2009. PMID: 19861460. DOI: 10.1158/1078-0432.CCR-09-0695 
27 Di Stefano L, Jensen MR and Helin K: E2F7, a novel E2F featuring DP-independent repression of a subset of E2Fregulated genes. EMBO J 22(23): 6289-6298, 2003. PMID: 14633988. DOI: $10.1093 / \mathrm{emboj} / \mathrm{cdg} 613$

28 Yin W, Wang B, Ding M, Huo Y, Hu H, Cai R, Zhou T, Gao Z, Wang $\mathrm{Z}$ and Chen D: Elevated E2F7 expression predicts poor prognosis in human patients with gliomas. J Clin Neurosci 33: 187193, 2016. PMID: 27460513. DOI: 10.1016/j.jocn.2016.04.019

29 Schneider M, Schneider S, Zühlke-Jenisch R, Klapper W, Sundström C, Hartmann S, Hansmann ML, Siebert R, Küppers $\mathrm{R}$ and Giefing M: Alterations of the CD58 gene in classical Hodgkin lymphoma. Genes Chromosomes Cancer 54(10): 638645, 2015. PMID: 26194173. DOI: $10.1002 / \mathrm{gcc} .22276$

30 Xu S, Wen Z, Jiang Q, Zhu L, Feng S, Zhao Y, Wu J, Dong Q, Mao $\mathrm{J}$ and Zhu Y: CD58, a novel surface marker, promotes self-renewal of tumor-initiating cells in colorectal cancer. Oncogene 34(12): 1520-1531, 2015. PMID: 24727892. DOI: 10.1038/onc.2014.95

31 Mäenpää A, Kovanen PE, Paetau A, Jäáskeläinen J and Timonen T: Lymphocyte adhesion molecule ligands and extracellular matrix proteins in gliomas and normal brain: expression of VCAM-1 in gliomas. Acta Neuropathol 94(3): 216-225, 1997. PMID: 9292690 . DOI: $10.1007 / \mathrm{s} 004010050696$
32 Harris T, Marquez B, Suarez S and Schimenti J: Sperm motility defects and infertility in male mice with a mutation in Nsun7, a member of the Sun domain-containing family of putative RNA methyltransferases. Biol Reprod 77(2): 376-382, 2007. PMID: 17442852. DOI: $10.1095 /$ biolreprod.106.058669

33 Khosronezhad N, Hosseinzadeh Colagar A and Mortazavi SM: The Nsun7 (A11337)-deletion mutation, causes reduction of its protein rate and associated with sperm motility defect in infertile men. J Assist Reprod Genet 32(5): 807-815, 2015. PMID: 25702163. DOI: $10.1007 / \mathrm{s} 10815-015-0443-0$
Received February 20, 2020

Revised April 7, 2020

Accepted April 10, 2020 